\title{
Higher Cerebral Blood Flow Predicts Early Hematoma Expansion in Patients With Intracerebral Hemorrhage: A Clinical Study
}

\author{
Weijing Wang ${ }^{1,2,3 \dagger}$, Weitao Jin ${ }^{4 \dagger}$, Hao Feng ${ }^{1,5,6}$, Guoliang Wu ${ }^{7}$, Wenjuan Wang ${ }^{1,5,6}$, \\ Jiaokun Jia ${ }^{1,5,6}$, Ruijun Ji ${ }^{1,5,6}$, Anxin Wang ${ }^{1}$ and Xingquan Zhao ${ }^{1,5,6 *}$
}

'Department of Neurology, Beijing Tiantan Hospital, Capital Medical University, Beijing, China, ${ }^{2}$ Department of Neurology, Beijing Haidian Hospital, Beijing, China, ${ }^{3}$ Department of Neurology, Haidian Section of Peking University Third Hospital, Beijing, China, ${ }^{4}$ Department of Neurosurgery, Peking University International Hospital, Beijing, China, ${ }^{5}$ China National Clinical Research Center for Neurological Diseases, Beijing, China, ${ }^{6}$ Research Unit of Artificial Intelligence in Cerebrovascular Disease, Chinese Academy of Medical Sciences, Beijing, China, ${ }^{7}$ Department of Radiology, Beijing Tiantan Hospital, Capital Medical University, Beijing, China

The early hematoma expansion of intracerebral hemorrhage $(\mathrm{ICH})$ indicates a poor prognosis. This paper studies the relationship between cerebral blood flow (CBF) around the hematoma and hematoma expansion (HE) in the acute stage of intracerebral hemorrhage. A total of 50 patients with supratentorial cerebral hemorrhage were enrolled in this study. They underwent baseline whole-brain CTP within $6 \mathrm{~h}$ after intracerebral hemorrhage, and non-contrast CT within $24 \mathrm{~h}$. Absolute hematoma growth and relative hematoma growth were calculated, respectively. A relative growth of Hematoma volume $>33 \%$ was considered to be hematoma expansion. The Ipsilateral peri-edema CBF and Ipsilateral edema CBF were calculated by CTP maps in patients with and without hematoma expansion, respectively. In this study the incidence of hematoma expansion in the early stage of supratentorial cerebral hemorrhage was $32 \%$; The CBF of the hematoma expansion group was higher than that of the patients without hematoma expansion ( $23.5 \pm 12.5$ vs. $15.1 \pm 7.4, P=0.004$ ). After adjusting for age, gender, Symptom onset to NCCT and Baseline hematoma volume, ipsilateral peri-edema CBF was still an independent risk factor for early $\mathrm{HE}$ (or $=1.095,95 \% \mathrm{Cl}=1.01-1.19$, $P=0.024)$. Here, we concluded that higher cerebral blood flow predicts early hematoma expansion in patients with intracerebral hemorrhage.

Keywords: intracerebral hemorrhage, hematoma expansion, cerebral blood flow (CBF), CT perfusion (CTP), prognosis

\section{INTRODUCTION}

Acute spontaneous (non-traumatic) intracerebral hemorrhage is the most common type of spontaneous intracerebral hemorrhage. It affects about 2 million people around the world every year and exhibits the worst prognosis of all stroke types (1). The burden of hemorrhagic stroke is increasing rapidly worldwide between 1990 and 2010, with an increase of $47 \%$ in the absolute number of people with incident hemorrhagic stroke, $20 \%$ in deaths, and $14 \%$ in DALYs (1).

Intracerebral hemorrhage (ICH) expansion occurs in about one-third of ICH patients and is strongly associated with a poor outcome $(2,3)$. 
Several imaging signs on non-contrast CT may be related to the hematoma expansion and poor prognosis after intracerebral hemorrhage (4-8). The spot sign on CT angiography also received attention as an indicator of the hematoma expansion (9). However, the interpretation of those signs in the CT relies on the clinical experience of neuroradiologists, and the standard of that interpretation is not objective and cannot be evaluated quantitatively.

In our study, CBF was used as a quantitative method to calculate the cerebral blood flow of edematous tissue around a hematoma and its surrounding tissue after early hemorrhage, so as to study the correlation between the cerebral blood flow and the hematoma expansion.

\section{METHODS}

\section{Study Design and Population Eligibility}

This study was a prospective, observational cohort study. From December 2014 to September 2016 patients who presented with an acute symptomatic and CT confirmed ICH were recruited to the study. Patients aged 18 years or older were eligible for entry. NCCT was performed in 215 patients who had acute symptoms (severe headache, paralysis, aphasia etc.). 184 patients were confirmed supratentorial intracerebral hemorrhage. 50 of them finished CTP within 6 hours and NCCT review within 24 hours after the symptoms attack. Therefore, 50 patients are included in this study (Figure 1).

Inclusion criteria: (1) age $>18$ years, (2) within $6 \mathrm{~h}$ of onset, (3) NCCT + CTA + CTP at baseline, (4) NCCT reexamination within $24 \mathrm{~h}$ of onset, (5) informed consent. Exclusion criteria: (1) failure to cooperate with the completion of imaging examination (refusal of examination or sensitization of imaging agent), (2) planned or completed surgical craniotomy or hematoma aspiration, (3) refusal to join the study.

\section{Sources of Funding}

This study was sponsored by Capital health research and development of special (grant number: Capital 2011-2004-03; institute: Beijing Municipal Commission of Health and Family Planning; the author who received the funding: XZ) and the Beijing Municipal Science \& Technology Commission, PR China (grant number: Z131107002213009).

\section{Image Acquisition}

All participants were scanned on a 16-slice multidetector CT (Somatom volume zoom; Siemens, Erlangen, Germany). Wholebrain NCCT was performed first with a slice thickness of $9 \mathrm{~mm}$ for the supratentorial area and $4.5 \mathrm{~mm}$ for the infratentorial area to confirm primary ICH. Acquisition parameters were: $120 \mathrm{kVp}$; $310 \mathrm{~mA}$; and field of view $(\mathrm{FOV})=24 \mathrm{~cm}(10)$.

CTP covering two continuous sections at the level across the maximum transverse section of the hematoma lesion was performed. The scanning parameters were: tube current $=80$ $\mathrm{kVp} ; 209 \mathrm{~mA}$; rotation time $1 / 41.0 \mathrm{~s} /$ rotation; total scan time $40 \mathrm{~s}$; section thickness $=12 \mathrm{~mm}$; and 40 images per section. CTP was started $4 \mathrm{~s}$ after injection of a bolus of $40 \mathrm{ml}$ of iobitridol (300 $\mathrm{mg} / \mathrm{mL}$, Xenetix; Guerbet, Aulnay-sous-Bois, France) at a rate of $8 \mathrm{~mL} / \mathrm{s}$ into the antecubital vein (with a 20-gauge intravenous

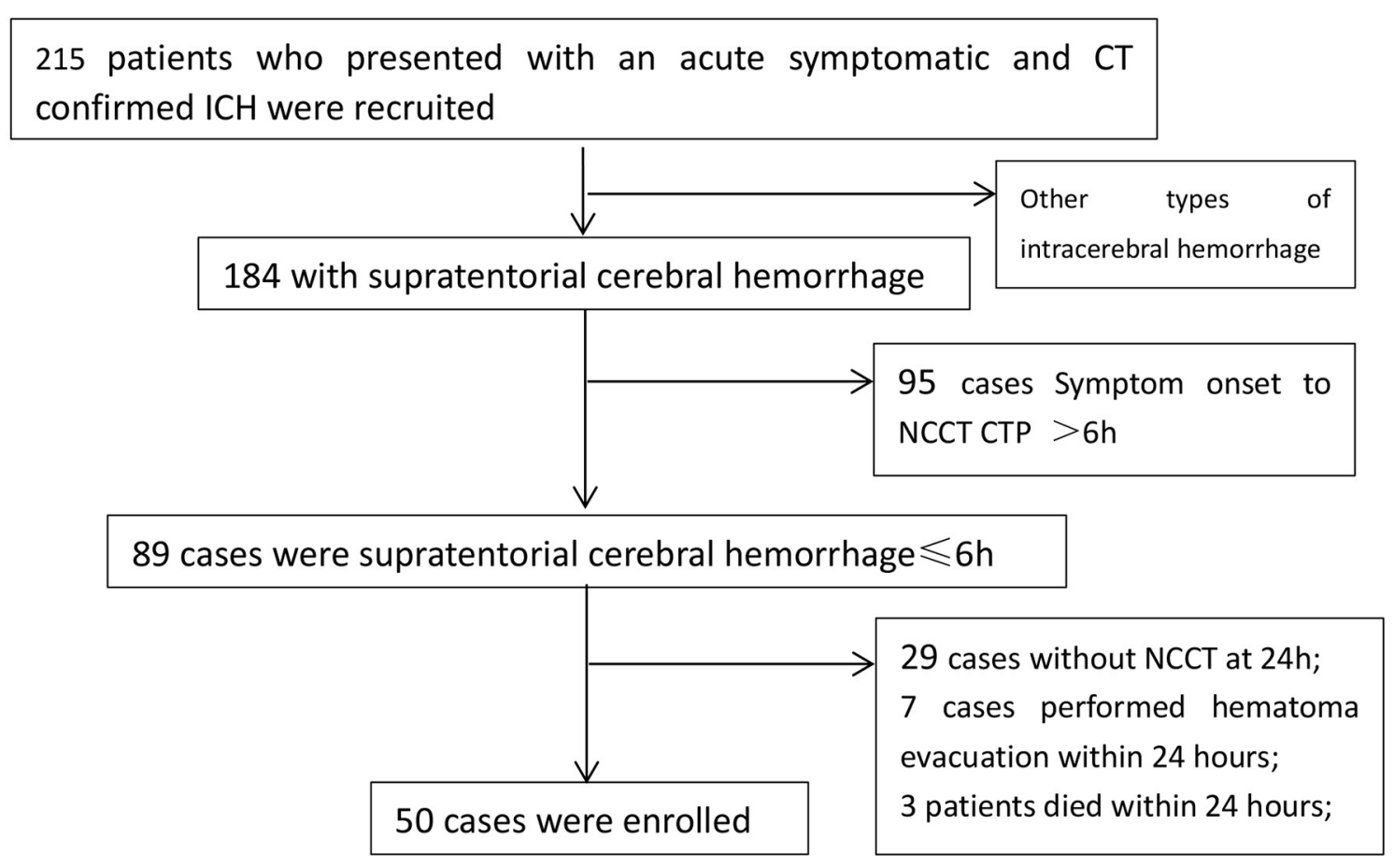

FIGURE 1 | Flow chart of patient selection. 

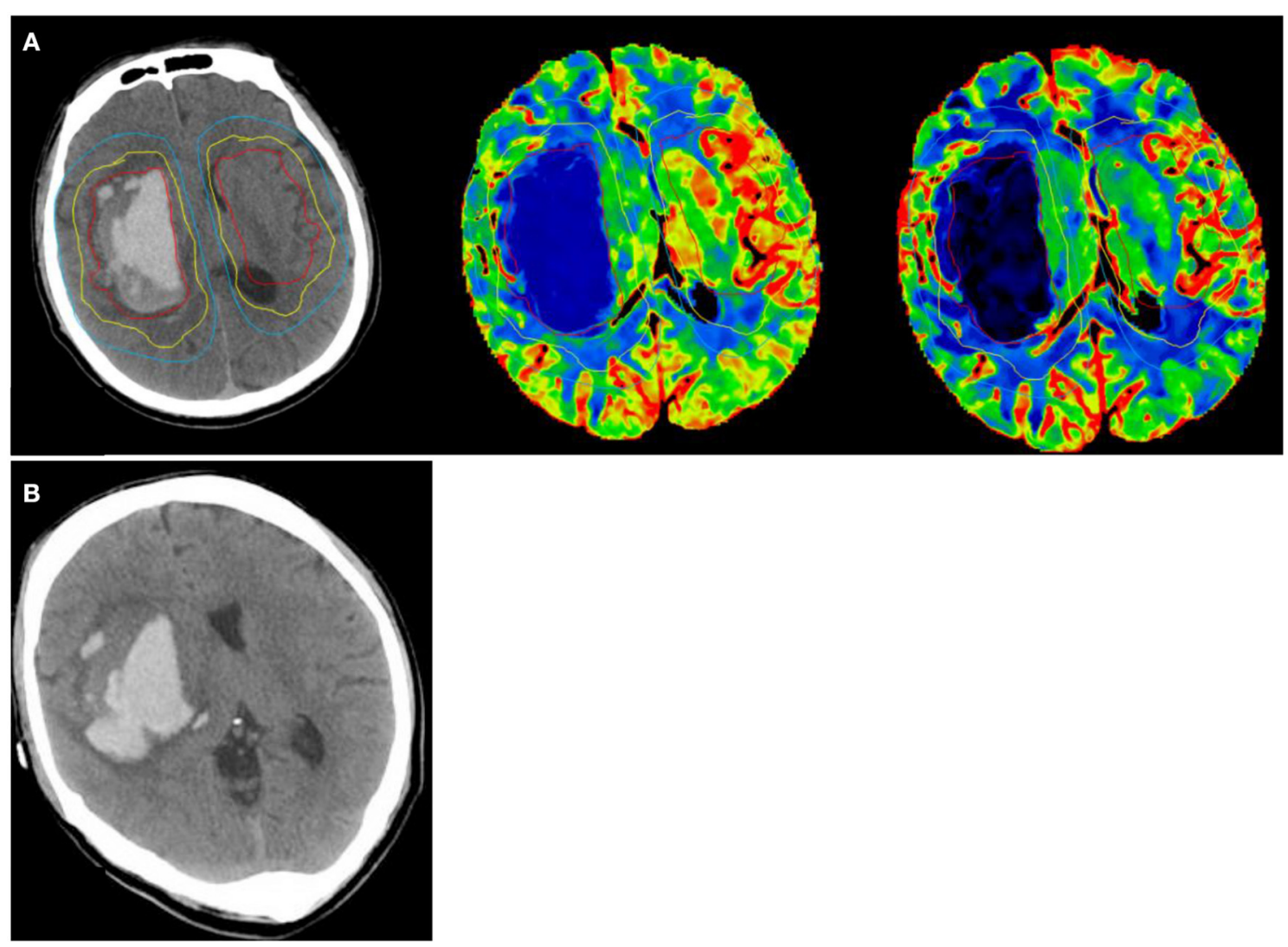

FIGURE 2 | A 42 year old man with a sudden headache and loss of consciousness, NCCT shows hematoma volume $49.5 \mathrm{ml}$ at randomization. Ipsilateral peri-edema CBF was $30.48 \mathrm{ml} / 100 \mathrm{~g} / \mathrm{min}$ at randomization. (A) (1) perihematomal low-density area in the yellow circle as Ipsilateral edema area; (2) an area mirroring the Ipsilateral edema area located in the contralateral hemisphere as Contralateral edema area; (3) $1 \mathrm{~cm}$ rim of normal-appearing brain tissue surrounding the perilesional low-density area in the blue circle as Ipsilateral peri-edema area; (4) an area mirroring the Ipsilateral peri-edema area located in the contralateral hemisphere as Contralateral peri-edema area. (B) NCCT at $24 \mathrm{~h}$ after symptom onset shows hematoma volume was $69.14 \mathrm{ml}$.

cannula) using a power injector. The effective radiation dose was $3.51 \mathrm{mSv}$ for one-time scanning (10).

All patients were followed up with NCCT using the same CT system and parameters $24 \mathrm{~h}$ after the onset of the disease to evaluate whether the hematoma expanded.

\section{Image Analysis}

Hematoma volumes were calculated at baseline and at followup NCCT images using the method as follows: Hematoma volumes were defined using semiautomated Hounsfield Unit thresholding. Edema volumes were measured using a threshold of 5 to 23 Hounsfield Units, which has been demonstrated to be the most reliable CT Hounsfield Unit threshold for edema $(11,12)$.

Absolute ICH growth (follow-up volume-baseline volume) and relative ICH growth ([follow-up volume-baseline volume]/baseline volume) were calculated, respectively. A relative hematoma increase growth $>33 \%$ was considered to indicate significant hematoma expansion (13). Post-processing of raw CTP source images was completed centrally on a GE aw workstation. CTP maps were derived from the tissue time-density curve and contrast bolus delay and dispersion were corrected for using a singular value deconvolution algorithm. Region of interest (ROI) analyses were completed using the Analyze 11.0 software package (13). As previously reported (14), ROIs were drawn using planimetric techniques on CTP base images and then transferred to the corresponding $\mathrm{CBF}, \mathrm{CBV}$, MTT maps. ROIs included the edema, a $1-\mathrm{cm}$ region surrounding the edema, contralateral mirror regions (Figure 2). Image post-processing was completed by an experienced neuroradiologist and then reviewed by a chief radiologist independently.

\section{Statistical Analysis}

Statistical analysis was performed using SPSS 21.0 (SPSS Inc, Chicago, IL). Comparison of baseline mean characteristics between the 2 groups was made using independent $t$-tests, Mann-Whitney tests, or Pearson $\chi^{2}$-tests. The relationships between hematoma expansion and CBF were assessed using Logistic regression. Paired $t$-tests were used to assess $\mathrm{CBF}$ differences between different parts. The influence of different 
TABLE 1 | Baseline characteristics.

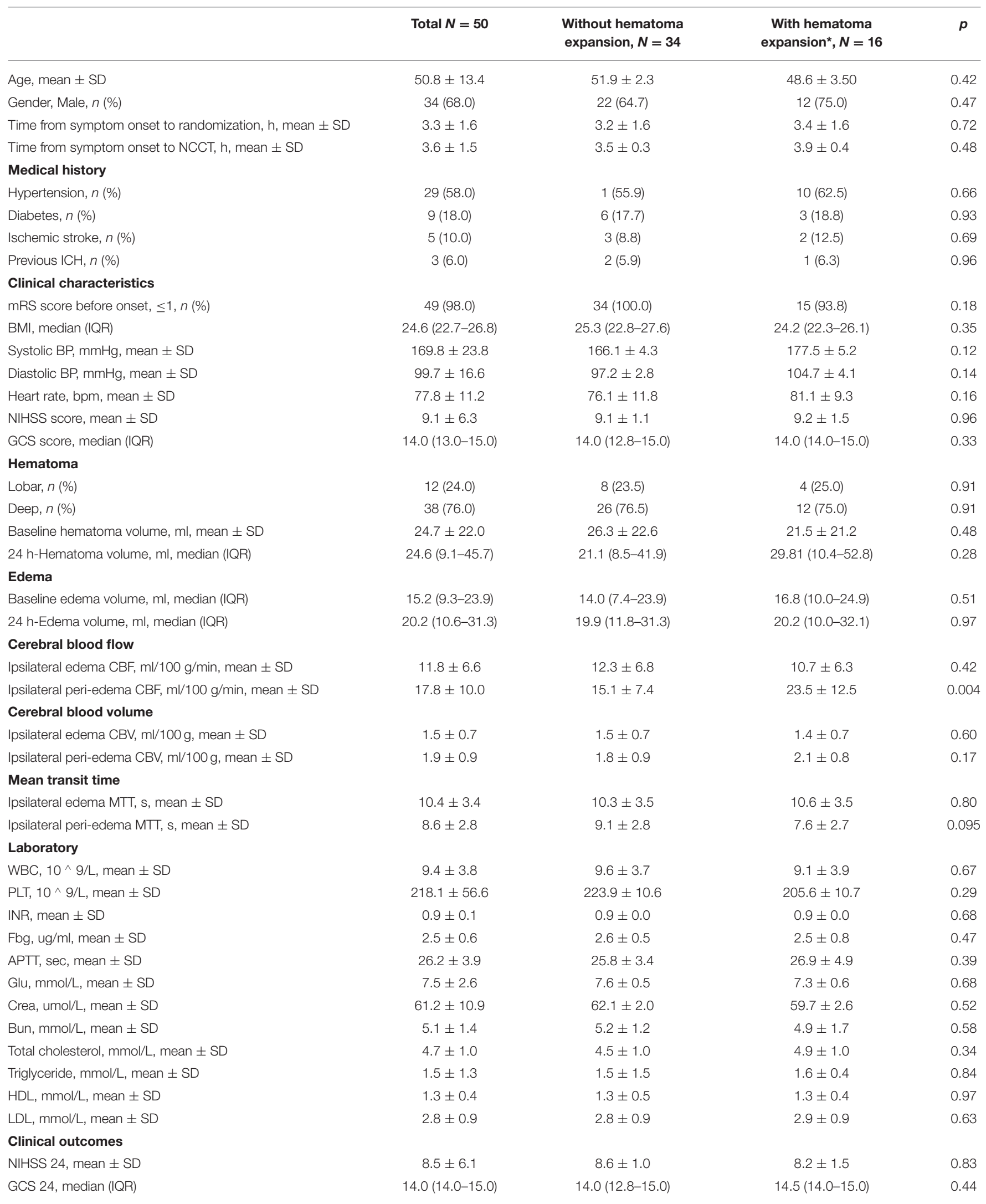

*Hematoma expansion defined as an absolute increase in hematoma $>12.5 \mathrm{ml}$ or relative growth $>33 \%$. 
TABLE 2 | CBF in different location.

\begin{tabular}{|c|c|c|c|}
\hline & $\begin{array}{l}\text { Ipsilateral } \\
\text { edema CBF, } \\
\mathrm{ml} / 100 \mathrm{~g} / \mathrm{min}\end{array}$ & $\begin{array}{c}\text { Ipsilateral } \\
\text { peri-edema CBF, } \\
\mathrm{ml} / 100 \mathrm{~g} / \mathrm{min}\end{array}$ & $p$ \\
\hline Total, $n=50$ & $11.80 \pm 6.64$ & $17.83 \pm 10.01$ & 0.001 \\
\hline $\begin{array}{l}\text { Without hematoma } \\
\text { expansion, } n=34\end{array}$ & $12.33 \pm 6.81$ & $15.14 \pm 7.40$ & 0.099 \\
\hline $\begin{array}{l}\text { With hematoma } \\
\text { expansion*, } n=16\end{array}$ & $10.67 \pm 6.33$ & $23.54 \pm 12.48$ & 0.002 \\
\hline
\end{tabular}

*Hematoma expansion defined as an relative increase in hematoma $>33 \%$.

sites and $\mathrm{CBF}$ on hematoma expansion was evaluated by the method of interaction analysis.

\section{RESULTS}

\section{Baseline Characteristics}

A total of 50 patients were included in the study (Baseline characteristics shown in Table 1). The study population included $34(68.0 \%)$ males and $16(32.0 \%)$ females with an average age of 50.8 years. The baseline demographic and clinical cohort characteristics are described in Table 1. The median time from symptom onset to baseline CT scan was $3.6 \pm 1.5 \mathrm{~h}$, The median baseline hematoma volume was $24.7 \pm 22.0 \mathrm{ml}$. 16 patients (32\%) showed early hematoma expansion. All patients completed CT Perfusion at baseline. There was a difference between the two groups in the CBF of the peri-edema area. (CBF on the peri-edema area of patients with hematoma expansion was 23.5 $\mathrm{ml} / 100 \mathrm{~g} / \mathrm{min}$; $\mathrm{CBF}$ on the peri-edema area of patients without hematoma expansion was $15.1 \mathrm{ml} / 100 \mathrm{~g} / \mathrm{min}$; $p$-value 0.004 ).

\section{CBF in Different Locations}

The mean Ipsilateral edema CBF was $11.8 \pm 6.6 \mathrm{ml} / 100 \mathrm{~g} / \mathrm{min}$. The mean Ipsilateral peri-edema CBF was $17.8 \pm 10.0 \mathrm{ml} / 100$ $\mathrm{g} / \mathrm{min}$. The Ipsilateral peri-edema CBF was higher than Ipsilateral edema CBF $(17.8 \pm 10.0 \mathrm{ml} / 100 \mathrm{~g} / \mathrm{min}$ vs. $11.8 \pm 6.6 \mathrm{ml} / 100$ $\mathrm{g} / \mathrm{min}, p=0.001)$ in all groups, the Ipsilateral peri-edema CBF was higher than Ipsilateral edema CBF $(15.1 \pm 7.4 \mathrm{ml} / 100 \mathrm{~g} / \mathrm{min}$ vs. $12.3 \pm 6.8 \mathrm{ml} / 100 \mathrm{~g} / \mathrm{min}, p=0.099)$ in patients without hematoma expansion group, and the Ipsilateral peri-edema $\mathrm{CBF}$ was higher than Ipsilateral edema CBF $(23.5 \pm 12.5 \mathrm{ml} / 100$ $\mathrm{g} / \mathrm{min}$ vs. $10.7 \pm 6.3 \mathrm{ml} / 100 \mathrm{~g} / \mathrm{min}, p=0.099)$ in patients with hematoma expansion group (Table 2).

\section{Multivariate Analysis of Hematoma Expansion}

Univariate analysis showed that Ipsilateral peri-edema CBF was a risk factor for early hematoma expansion (OR $=1.10,95 \%$ CI $1.02-1.19, P=0.014)$. Model 1 included covariates with $p<0.20$ in univariable analysis. Model 2 adjusted for age and sex. Model 3 adjusted for age, sex, and predictors of hemorrhage growth identified from the literature such as time from onset to NCCT and ICH volume. Multivariate analysis showed that Ipsilateral peri-edema $\mathrm{CBF}$ was an independent risk factor for hematoma expansion (Table 3 ).
TABLE 3 | Multivariate analysis of hematoma expansion.

\begin{tabular}{llccc}
\hline & & OR & OR (95\% Cl) & $\boldsymbol{p}$ \\
\hline Model 1 & SBP & 1.009 & $0.971-1.048$ & 0.655 \\
& DBP & 1.031 & $0.973-1.092$ & 0.303 \\
& Ipsilateral peri-edema CBF & 1.101 & $1.022-1.185$ & 0.011 \\
Model 2 & SBP & 1.013 & $0.973-1.054$ & 0.541 \\
& DBP & 1.030 & $0.971-1.092$ & 0.330 \\
& Ipsilateral peri-edema CBF & 1.260 & $1.022-1.544$ & 0.031 \\
& Ipsilateral peri-edema CBV & 0.288 & $0.050-1.661$ & 0.164 \\
& Ipsilateral peri-edema MTT & 1.336 & $0.859-2.079$ & 0.199 \\
Model 3 & Age & 1.010 & $0.953-1.070$ & 0.749 \\
& Gender (male) & 2.569 & $0.446-14.799$ & 0.291 \\
& SBP & 1.011 & $0.970-1.054$ & 0.595 \\
& DBP & 1.033 & $0.972-1.099$ & 0.296 \\
& Ipsilateral peri-edema CBF & 1.091 & $1.011-1.178$ & 0.025 \\
Model 4 & Age & 1.010 & $0.949-1.075$ & 0.754 \\
& Gender (male) & 4.447 & $0.556-35.543$ & 0.159 \\
& SBP & 1.017 & $0.974-1.061$ & 0.452 \\
& DBP & 1.032 & $0.970-1.098$ & 0.315 \\
& Ipsilateral peri-edema CBF & 1.095 & $1.012-1.185$ & 0.024 \\
& Contralateral peri-edema CBF & 1.031 & $0.949-1.120$ & 0.476 \\
& Symptom onset to NCCT (h) & 1.293 & $0.754-2.216$ & 0.350 \\
& Baseline hematoma volume (ml) & 0.983 & $0.945-1.021$ & 0.370 \\
\hline & & & &
\end{tabular}

Model 1: unadjusted.

Model 2: Adjusted CBV and MTT.

Model 3: Adjusted age and gender.

Model 4: Adjusted age, gender, Symptom onset to NCCT, and Baseline hematoma volume.

\section{DISCUSSION}

The incidence of hematoma expansion in the early stage of supratentorial intracerebral hemorrhage is $32 \%$ in this study. Hematoma expansion was defined as a $33 \%$ increase in hematoma volume compared with baseline (13), which is consistent with the results Brott et al. (15) reported in the previous literature of about $38 \%$. However, due to the disunity of the definitional standard of hematoma expansion, the incidence of hematoma expansion reported in the relevant studies is quite different. The longer the difference between the first CT and the reexamination $\mathrm{CT}$ is, the closer the first $\mathrm{CT}$ is to the onset time, and the incidence of hematoma expansion is correspondingly increased. In 1990, Fujitsu et al. (16) studied 107 patients with intracerebral hemorrhage within $6 \mathrm{~h}$ of onset, rescanned NCCT within $24 \mathrm{~h}$, and found that the incidence of hematoma expansion was about 20.3\%; Mayer et al. (17) observed 46 patients with intracerebral hemorrhage within $11 \mathrm{~h}$ of onset, and the hematoma expansion rate was only $9 \%$.

In recent years, some clinical studies have focused on the relationship between hematoma expansion and prognosis. Some results have revealed the relationship between hematoma expansion and poor prognosis. Davis et al. (18) in a 2006 meta-analysis showed that hematoma volume increased by $10 \%$, mortality increased by $5 \%$. 
However, the pathophysiological mechanism of hematoma expansion is still unclear. Further study on the pathophysiological mechanism of hematoma expansion and the cerebral blood flow around the hematoma is helpful to clarify the causes of hematoma expansion, so as to provide targeted prevention and treatment and improve the prognosis.

This study showed the CBF of ipsilateral peri-edema was higher than that of edema CBF, suggesting that after cerebral hemorrhage, the edema was hypoperfusion, and there was an area of hyperperfusion around the edema. Using single-photon emission computed tomography (SPECT), Mayer et al. (19) demonstrated depressed perfusion surrounding the hemorrhage and concluded that the restoration of perilesional blood flow in the early stage of $\mathrm{ICH}$ resulted from perihematoma cerebral edema formation. Zhou et al. (20) found that a gradient of hypoperfusion surrounding the hematoma, which showed a thin rim of reduced perfusion in the perilesional zone in rCBF maps. In addition to perilesional hyperperfusion, the local hyperemia can also be observed in the regions distant from the hematoma, even in the cortical regions of the uninvolved hemisphere. The hyperemia often results from the vasodilation of pia arteries and arterioles in the periphery of the injury zone (19), as efforts are made to restore blood flow in the regions of reduced perfusion after ICH onset. The unstable blood flow perfusion occurring in perilesional tissue and remote brain may, on the one hand, reflect the exhaustion of autoregulation of CBF correlated with $\mathrm{ICH}$, and on the other hand, predict that the secondary brain injury will occur in the related cerebral tissue.

Intracerebral hemorrhage is a dynamic, complex, and continuous process. Hematoma expansion occurs in the early stage of intracerebral hemorrhage. The hematoma expansion comes from blood pressure. The higher the blood pressure is, the more likely the hematoma to expand. There are two reasons why the hematoma is no longer enlarged. One is the spontaneous hemostasis mechanism after vascular rupture; the other is that the pressure inside and outside the ruptured blood vessel wall is reduced due to the pressure increase in the hematoma cavity caused by edema compression. Therefore, the study of edema area and surrounding tissue is helpful to understand the pathophysiological mechanism of hematoma expansion.

On the other hand, some imaging signs can also indicate the occurrence of hematoma expansion. The most classic is the "spot sign" (9). A spot sign is defined as one or more 1to 2-mm foci of enhancement within the hematoma on CTA source images, which is the exudation point of contrast inside the hematoma. The occurrence of spot signs indicates an increased risk of hematoma expansion. The spot signs indicated that the enlargement of the hematoma was caused by the internal part of the hematoma.

However, some researchers have found that island sign can also indicate the risk of hematoma expansion (8). Island sign is the irregular edge of hematoma, suggesting the occurrence of hematoma expansion, which is caused by the fact that some relatively normal tissues outside the hematoma also become hemorrhagic brain tissue.

This study showed that there were blood flow changes in the peripheral tissue of hematoma. The higher the CBF of the tissue at the far side of the edema, the more likely the hematoma to expand, suggesting that the hematoma expansion may start from the outside.

However, the exact pathophysiological mechanism of hematoma expansion remains to be confirmed, for patients with ICH who have completed CTP examination in the early stage of admission, the measurement of ipsilateral peri edema CBF can be used as a supplementary prediction tool.

\section{STUDY LIMITATION}

In our study, due to the strict limitations of timing and sequence of imaging examination, only 50 patients met the inclusion criteria, and more than 150 patients were excluded, which may have resulted in a selective bias. On the other hand, the patients who underwent NCCT and CTP at baseline and reviewed NCCT $24 \mathrm{~h}$ usually need to be in a relatively stable condition, and so for the patients with a large or deadly hemorrhage, whether the conclusion is still applicable is not clear. This study found that the relative increased $\mathrm{rCBF}$ between the peri-hematoma region and the contralateral region related with the hemorrhage expansion, but the increase of the $\mathrm{CBF}$ in peri-hematoma region did not show a similar result. This could be due to an interference caused by the treatments such as lowering the blood pressure or use of mannitol. These treatments could reduce the CBF of both sides, but the different vascular reactivity between the tissues surrounding the hematoma and the contralateral side made the range of decrease different, and then impacted on the result. However, this assumption still requires further study to be confirmed.

\section{CONCLUSION}

Higher cerebral blood flow predicts early hematoma expansion in patients with intracerebral hemorrhage.

\section{DATA AVAILABILITY STATEMENT}

The original contributions presented in the study are included in the article/supplementary material, further inquiries can be directed to the corresponding author/s.

\section{ETHICS STATEMENT}

The studies involving human participants were reviewed and approved by Ethics Committee of Tiantan Hospital. The patients/participants provided their written informed consent to participate in this study.

\section{AUTHOR CONTRIBUTIONS}

WeiW and $\mathrm{XZ}$ contributed to the conception and design of the study. WenW and JJ organized the database. HF and GW performed the statistical analysis. WeiW and WJ wrote the first draft of the manuscript. RJ and AW revised the manuscript. All authors contributed to manuscript revision, read, and approved the submitted version. 


\section{FUNDING}

This study was sponsored by Capital Health Research and Development of Special (Grant No. Capital 2011-2004-03;

\section{REFERENCES}

1. Krishnamurthi RV, Feigin VL, Forouzanfar MH, Mensah GA, Connor M, Bennett DA, et al. Global and regional burden of first-ever ischaemic and haemorrhagic stroke during 1990-2010: findings from the global burden of disease study 2010. Lancet Glob Health. (2013) 1:e25981. doi: 10.1016/S2214-109X(13)70089-5

2. Dowlatshahi D, Demchuk AM, Flaherty ML, Ali M, Lyden PL, Smith EE. Defining hematoma expansion in intracerebral hemorrhage: relationship with patient outcomes. Neurology. (2011) 76:1238-44. doi: 10.1212/WNL.0b013e3182143317

3. Steiner $\mathrm{T}$, Bösel J. Options to restrict hematoma expansion after spontaneous intracerebral hemorrhage. Stroke. (2010) 4::402-9. doi: 10.1161/STROKEAHA.109.552919

4. Barras CD, Tress BM, Christensen S, MacGregor L, Collins M, Desmond PM, et al. Density and shape as CT predictors of intracerebral hemorrhage growth. Stroke. (2009) 40:1325-31. doi: 10.1161/STROKEAHA.108.536888

5. Blacquiere D, Demchuk AM, Al-Hazzaa M, Deshpande A, Petrcich W, Aviv $\mathrm{RI}$, et al. Intracerebral hematoma morphologic appearance on noncontrast computed tomography predicts significant hematoma expansion. Stroke. (2015) 46:3111-6. doi: 10.1161/STROKEAHA.115.010566

6. Li Q, Zhang G, Huang YJ, Dong MX, Lv FJ, Wei X, et al. Blend sign on computed tomography: novel and reliable predictor for early hematoma growth in patients with intracerebral hemorrhage. Stroke. (2015) 46:211923. doi: 10.1161/STROKEAHA.115.009185

7. Li Q, Zhang G, Huang YJ, Dong MX, Lv FJ, Wei X, et al. Black hole sign: novel imaging marker that predicts hematoma growth in patients with intracerebral hemorrhage. Stroke. (2016) 47:1777-81. doi: 10.1161/STROKEAHA.116.013186

8. Li Q, Zhang G, Huang YJ, Dong MX, Lv FJ, Wei X, et al. Island sign: an imaging predictor for early hematoma expansion and poor outcome in patients with intracerebral hemorrhage. Stroke. (2017) 48:301925. doi: 10.1161/STROKEAHA.117.017985

9. Wada R, Aviv RI, Fox AJ, Sahlas DJ, Gladstone DJ, Tomlinson $\mathrm{G}$, et al. CT angiography "spot sign" predicts hematoma expansion in acute intracerebral hemorrhage. Stroke. (2007) 38:1257-62. doi: 10.1161/01.STR.0000259633.59404.f3

10. Fu F, Sui B, Liu L, Su Y, Sun S, Li Y. Quantitative assessment of local perfusion change in acute intracerebral hemorrhage areas with and without “dynamic spot sign" using CT perfusion imaging. Acta Radiol. (2019) 60:36773. doi: 10.1177/0284185118780893

11. McCourt R, Gould B, Gioia L, Kate M, Coutts SB, Dowlatshahi D, et al. Cerebral perfusion and blood pressure do not affect perihematoma edema growth in acute intracerebral hemorrhage. Stroke. (2014) 45:12928. doi: 10.1161/STROKEAHA.113.003194

12. Clasen RA, Huckman MS, Von Roenn KA, Pandolfi S, Laing I, Lobick JJ. A correlative study of computed tomography and histology in human and experimental vasogenic cerebral edema. J Comput Assist Tomogr. (1981) 5:313-27. doi: 10.1097/00004728-198106000-00001
Institute: Beijing Municipal Commission of Health and Family Planning; the author who received the funding: XZ) and the Beijing Municipal Science \& Technology Commission, PR China (Grant No. Z131107002213009).

13. Kazui S, Naritomi H, Yamamoto H, Sawada T, Yamaguchi T. Enlargement of spontaneous intracerebral hemorrhage. Incidence and time course. Stroke. (1996) 27:1783-7. doi: 10.1161/01.STR.27.10.1783

14. Fainardi E, Borrelli M, Saletti A, Schivalocchi R, Azzini C, Cavallo M, et al. CT perfusion mapping of hemodynamic disturbances associated to acute spontaneous intracerebral hemorrhage. Neuroradiology. (2008) 50:72940. doi: 10.1007/s00234-008-0402-x

15. Brott T, Broderick J, Kothari R, Barsan W, Tomsick T, Sauerbeck L, et al. Early hemorrhage growth in patients with intracerebral hemorrhage. Stroke. (1997) 28:1-5. doi: 10.1161/01.STR.28.1.1

16. Egila AA, Khodair RM, Faheem MK, Kasem SM. Hematoma expansion in spontaneous intracerebral hemorrhage. J neurosurg. (1994) 80:517. doi: 10.3171/jns.1994.80.1.0051

17. Fujitsu K, Muramoto M, Ikeda Y, Inada Y, Kim I, Kuwabara T. Indications for surgical treatment of putaminal hemorrhage. Journal of Neurosurgery. (2009) 73:518-25. doi: 10.3171/jns.1990.73.4.0518

18. Davis SM, Broderick J, Hennerici M, Brun NC, Diringer $\mathrm{MN}$, Mayer SA, et al. Hematoma growth is a determinant of mortality and poor outcome after intracerebral hemorrhage. Neurology. (2006) 66:1175-81. doi: 10.1212/01.wnl.0000208408.984 82.99

19. Mayer SA, Lignelli A, Fink ME, Kessler DB, Thomas CE, Swarup R, et al. Perilesional blood flow and edema formation in acute intracerebral hemorrhage: a SPECT study. Stroke. (1998) 29:1791-8. doi: 10.1161/01.STR.29.9.1791

20. Zhou J, Zhang H, Gao P, Lin Y, Li X. Assessment of perihematomal hypoperfusion injury in subacute and chronic intracerebral hemorrhage by CT perfusion imaging. Neurol Res. (2010) 32:642-9. doi: 10.1179/016164109X124456165 96328

Conflict of Interest: The authors declare that the research was conducted in the absence of any commercial or financial relationships that could be construed as a potential conflict of interest.

Publisher's Note: All claims expressed in this article are solely those of the authors and do not necessarily represent those of their affiliated organizations, or those of the publisher, the editors and the reviewers. Any product that may be evaluated in this article, or claim that may be made by its manufacturer, is not guaranteed or endorsed by the publisher.

Copyright (C) 2021 Wang, Jin, Feng, Wu, Wang, Jia, Ji, Wang and Zhao. This is an open-access article distributed under the terms of the Creative Commons Attribution License (CC BY). The use, distribution or reproduction in other forums is permitted, provided the original author(s) and the copyright owner(s) are credited and that the original publication in this journal is cited, in accordance with accepted academic practice. No use, distribution or reproduction is permitted which does not comply with these terms. 